\title{
A CASE STUDY OF PEDESTRIAN POSITIONING WITH UWB AND UAV CAMERAS
}

\author{
A. Masiero ${ }^{1 *}$ P. Dabove ${ }^{2}$, V. Di Pietra ${ }^{2}$, M. Piragnolo ${ }^{3}$, A. Vettore ${ }^{3}$, S. Cucchiaro ${ }^{3}$, A. Guarnieri $^{3}$, P. Tarolli ${ }^{3}$, \\ C. Toth $^{4}$, V. Gikas ${ }^{5}$, H. Perakis ${ }^{5}$, K.-W. Chiang ${ }^{6}$, L.M. Ruotsalainen ${ }^{7}$, S. Goel ${ }^{8}$, J. Gabela ${ }^{9}$ \\ ${ }^{1}$ University of Florence, Italy - andrea.masiero@unifi.it \\ ${ }^{2}$ Politecnico di Torino, Italy - (paolo.dabove, vincenzo.dipietra)@ polito.it \\ ${ }^{3}$ University of Padova, Italy - (marco.piragnolo, antonio.vettore, sara.cucchiaro, alberto.guarnieri, paolo.tarolli)@unipd.it \\ ${ }^{4}$ Ohio State University, US - toth.2@ osu.edu \\ 5 National Technical University of Athens (NTUA), Greece - (vgikas, hperakis)@ central.ntua.gr \\ ${ }^{6}$ National Cheng Kung University, Tainan, Taiwan - kwchiang@ mail.ncku.edu.tw \\ ${ }^{7}$ University of Helsinki, Finland - laura.ruotsalainen@ helsinki.fi \\ 8 Indian Institute of Technology Kanpur, India - sgoel@iitk.ac.in \\ ${ }^{9}$ RMIT University, Australia - jelena.gabela@ rmit.edu.au
}

Commission I, WG I/7

KEY WORDS: Pedestrian positioning, UWB, Vision, GNSS-denied environments, UAV.

\begin{abstract}
:
The development and availability on consumer devices of the global navigation satellite systems (GNSS) have dramatically changed the everyday-life of most of the human population, enabling real time navigation on almost any smart device produced in the last years. However, such strong dependence on the availability of the GNSS limits the further spread of location based services to the areas where GNSS is not available or reliable. Motivated by these considerations, several research groups recently considered the problem of developing alternative positioning systems able to compensate the unavailability of GNSS in certain areas. Similarly, this paper also investigates the performance of certain alternative methods, which aim at partially substitute GNSS. In particular, the positioning performance of an affordable Ultra Wide-Band system is compared with that of a vision approach, based on the use of visual information acquired by an Unmanned Aerial Vehicle (UAV) flying over the area of interest. In accordance with the results obtained in our dataset, the drone-based vision system usually allow to obtain better positioning results when the pedestrians are visible in the drone video frames (median 2D positioning error less than $25 \mathrm{~cm}$ ). Nevertheless, the combination of such strategies shall also be investigated in order to obtain a more robust positioning system.
\end{abstract}

\section{INTRODUCTION}

Since the initial availability of the Global Navigation Satellite Systems (GNSS) for civilian use, the number of applications exploiting global position information has grown dramatically. As a matter of fact, the availability of a reliable positioning system, able to properly locate objects/persons of interest, can be considered a technology enabling the development of many applications and services, involving many sectors, ranging from transportation to geomatics and pedestrian positioning. GNSS currently play a dominant role in the positioning system scene: 1.7 billion GNSS devices were shipped in 2019, making smartphones the most used GNSS-enabled electronic device worldwide (GSA, 2019). Besides, wearables represent the beginning of the separation between smartphones and endusers (GSA, 2019). An increasing number of smartphone services and apps are now accessible via new interfaces (smartwatches, fitness trackers, smart glasses, clothing, etc.). Nevertheless, the need for availability, accuracy, continuity and integrity of pedestrian positioning and navigation systems in challenging GNSS environments is still increasing (de Groot et al., 2018)(Hsu et al., 2015)(Zeng et al., 2017), even in real-time (Dabove and Di Pietra, 2019). One of the main application fields is the Location Based Services (LBS) in urban environments (Luo et al., 2021), where GNSS is the main technique employed even considering portable devices, such as smart- phones and tablets. Despite the hardware limitations associated with low-cost devices, worldwide GNSS sales are largely dominated by this kind of devices. Indeed, such devices can be used in a multitude of location-aware applications. However, their success is also stimulating an increasing quest for systems able to go beyond the current limitations of low-cost GNSS devices. Consequently, the request for reliable, ubiquitously available and accurate positioning and navigation systems motivates the need to improve the current state of the art of lowcost GNSS positioning systems, for instance, taking advantage of new positioning approaches of other sensors (Retscher and Leb, 2021) that are often already embedded in users devices. In this context, the goal is to increase the accuracy and extend the usability areas of reliable positioning on consumer devices (e.g. smartphones, wearable devices). Ubiquitous seamless positioning, including both indoor and outdoor positioning and navigation, is one of the target applications in this case (Alinsavath et al., 2019, Retscher and Kealy, 2006). Since smartphones are provided with several sensors, integrating the information provided by such sensors is commonly agreed within the research community as a potentially viable way to achieve the desired ubiquitous positioning system (Gabela et al., 2019, Retscher et al., 2020, Sturari et al., 2016).

\footnotetext{
* Corresponding author
} 


\section{SCENARIO AND GOAL}

In accordance with the above mentioned request for improving the performance of consumer device positioning systems in conditions critical for GNSS, e.g. in urban canyons, this paper is part of a research project aiming at investigating the development of a positioning system based on sensors embedded in smartphones and on the potential usefulness of certain external infrastructures, such as Ultra Wide-Band (UWB) static networks and cameras.

For what concerns the use of UWB, it is worth to notice that certain of the recently commercialized smartphones are already equipped with UWB transceivers.

Furthermore, in addition to cameras embedded in the smartphones, even cameras installed in the area of interest (either ad hoc, or for other purposes, e.g. monitoring and surveillance, if they are enabled to communicate with the pedestrian devices) can be useful to such aim.

To mimic the above mentioned scenario, and evaluate also the general case in which drones may be available as well, the following working conditions have been considered in a measurement campaign jointly conducted by the ISPRS WG I/7 and the IAG WG 4.1.4 on October 21, 2020:

- Three pedestrians walked for approximately 20 minutes in the test area. Each pedestrian was provided with a smartphone, an Emlid GNSS receiver (Emlid Ltd, 2019), and a Pozyx UWB transceiver (Pozyx Labs, 2015). The smartphones acquired inertial data, GNSS raw measurements, and video with the (main) rear camera during all the test duration. Fig. 1 shows the three pedestrians during the test.

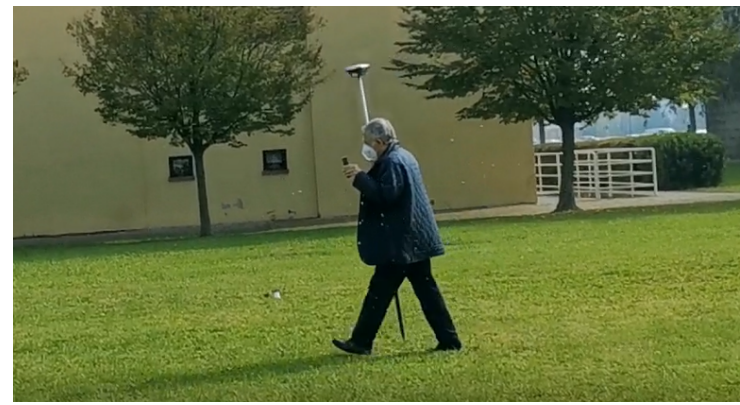

(a)

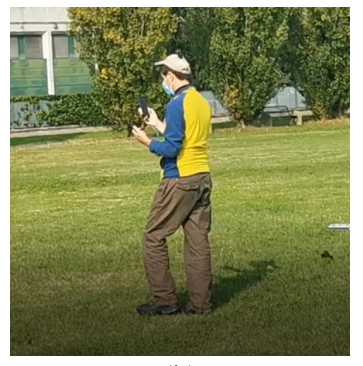

(b)

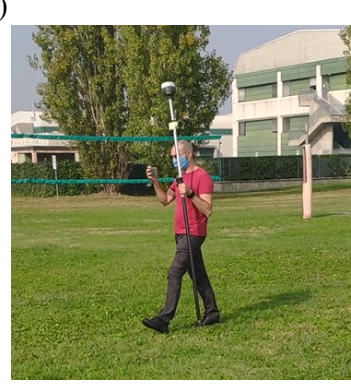

(c)
Figure 1. The three pedestrians during the data acquisition.

- A static UWB infrastructure have been deployed, corresponding to eight UWB Pozyx anchors distributed over all the area of interest (Fig. 2).

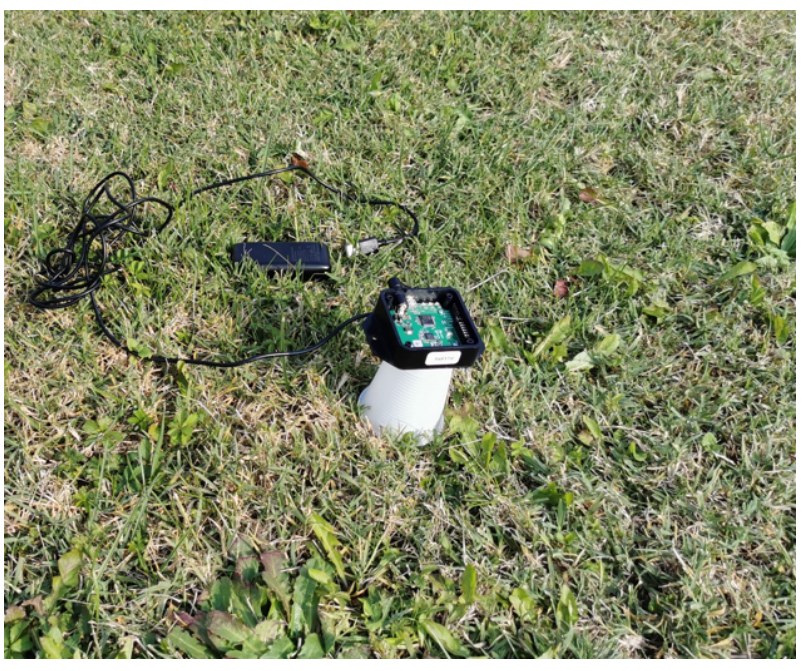

Figure 2. Example of Pozyx UWB anchor.

- Two drones, provided with Emlid GNSS receivers and Pozyx UWB transceivers, flew over the area, filming the pedestrian movements from two different points of view (e.g. nadir and oblique views, see Fig. 3). The sensors attached to one of the two drones, a DJI Matrice 210, can be seen in Fig. 4.

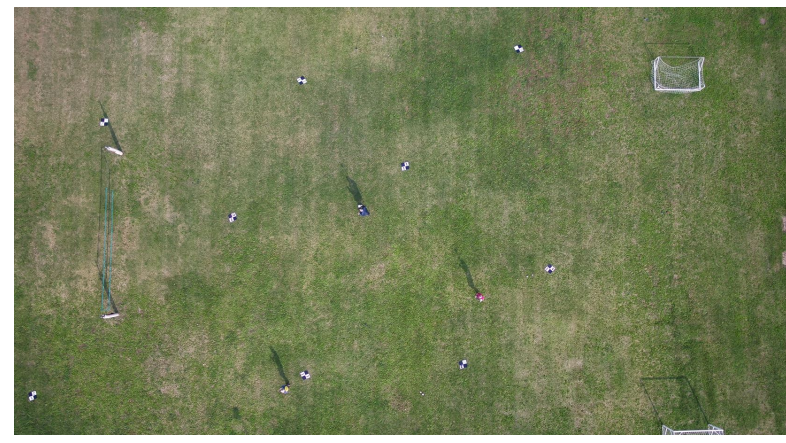

(a)

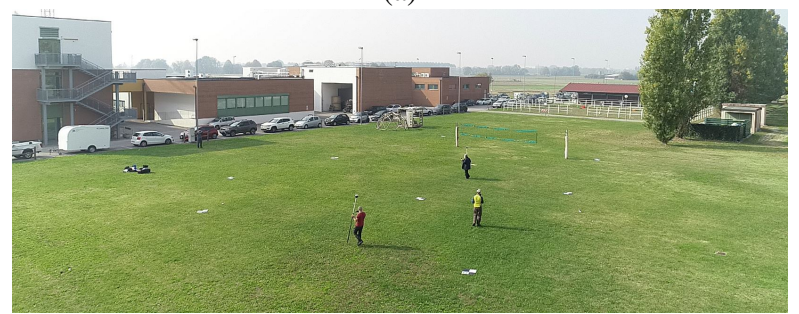

(b)

Figure 3. Drone views during the test: (a) DJI Matrice 210 nadir view, (b) DJI Phantom 4 Pro oblique view.

A set of targets has also been distributed in the area, and their positions has been measured by means of standard surveying tools.

The test area, in the Agripolis Campus of the University of Padua, has been chosen in such a way to ensure properly working conditions for the GNSS receivers (working in postprocessing kinematic, exploiting raw measurements from a permanent station at less than 100 meters from the test area), which hence can be used to retrieve reference trajectories, and the safety of persons in the Campus. 


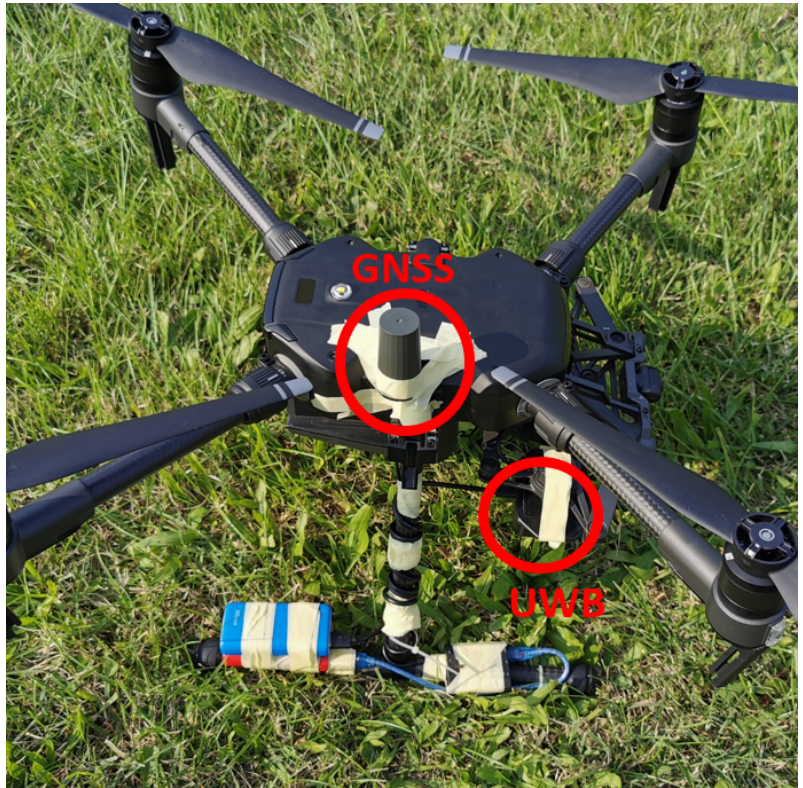

Figure 4. GNSS and UWB external sensors attached to DJI Matrice 210 drone.

This paper compares the performance of two alternative positioning approaches, i.e. based on static UWB terrestrial infrastructure, and on drone vision.

\section{METHODS}

The UWB positioning approach, based on a static infrastructure is similar to the one already developed by some of the authors in one of their previous works (Gabela et al., 2019). Hence, the reader is referred to (Gabela et al., 2019) for a detailed description of the UWB-based positioning approach. It is worth to notice that a different communication strategy between the UWB devices can be considered as well, e.g. letting the UWB network be adaptable to the set of really available devices: in such case an ad-hoc network may be implemented (Sakr et al., 2020).

The rest of this section is dedicated to provide some details on the developed drone vision-based positioning method.

First, the drone was mostly static and stable during the data collection, hence the recorded video frames refer to almost the same area.

Then, the movement of any frame with respect to the previous one (or with respect to a conventional one) is computed by means of a standard feature-based approach:

- BRISK (Leutenegger et al., 2011) feature descriptors are computed an matched

- The relative pose between two frames is estimated by means of a RANSAC procedure (Fischler and Bolles, 1981). It is also worth to notice that, since the camera is assumed to be stabilized by means of a proper gimbal, and (for instance) oriented in the nadir direction, the relative pose estimation problem can be reduced to the estimation of a 2D translation and rotation (He and Habib, 2016). Despite best results can be obtained correcting the lens distortion as well, in this work distortion has been discarded.
Thanks to such relative pose estimation, any frame can be remapped according to the orientation and position of a previous one.

Pedestrian detection on the video frames can be effectively implemented by means of a background subtraction approach: first, a background model is computed by considering the statistics of the pixel values on any location for a certain time interval (some hundreds of frames are usually quite sufficient for computing a decent statistical model of the pixel color variation), where pixel locations are remapped according to the previously estimated relative motion. A threshold value on each pixel is then used to detect the pixels potentially describing a moving person.

Then a box filter and an area threshold are applied in order to select only those areas where a certain amount of pixels have been detected has changed with respect to the computed background. The local maxima of the box filter outcome are considered as the positions associated to the centroids of pedestrians.

Since the such workflow is iteratively applied at each frame, the previous position of a pedestrian is used to limit the searching area of the new pedestrian position, which is supposed to be quite close to the previous one.

Furthermore, when two or more pedestrians are very close to each other the measurements on a frame with their real positions may be quite difficult. In such case, and also when a pedestrian shall be detected again after having moving out of the area visible in the video frame, a normalized correlation approach is used for properly associating any detected area representing a foreground subject to the correct pedestrian. To such aim, templates, such as those in Fig. 5, are used to compute the normalized correlations. Since the pedestrian can change its heading direction during the test, the normalized correlations are computed with different rotated versions of the templates, and only the maximum outcome is considered.
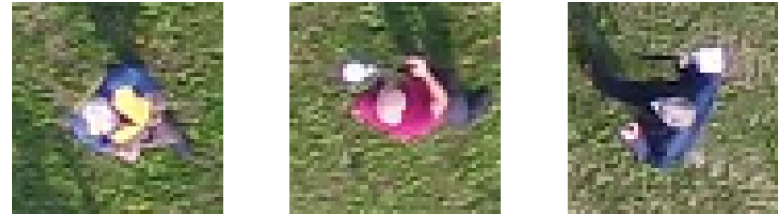

Figure 5. Top view of the pedestrians (from a drone video frame).

Once the pedestrians are detected on a video frame, their real position shall be computed. To such aim, a proper local map between the image and the real pedestrian coordinates is established by exploiting the target positions, both the real ones and those in at least one video frame, which are assumed to be known.

\section{RESULTS}

Figure 6 shows an example of UWB based positioning results: the figure compares the GNSS-based reference trajectory of a pedestrian (blue), with the one estimated by means of the static UWB infrastructure (UWB anchors are shown as black circular marks).

Despite the results shown in Figure 6 are quite decent in the area included within the UWB anchors, the positioning system had some flaws when the pedestrian went outside of such area. 


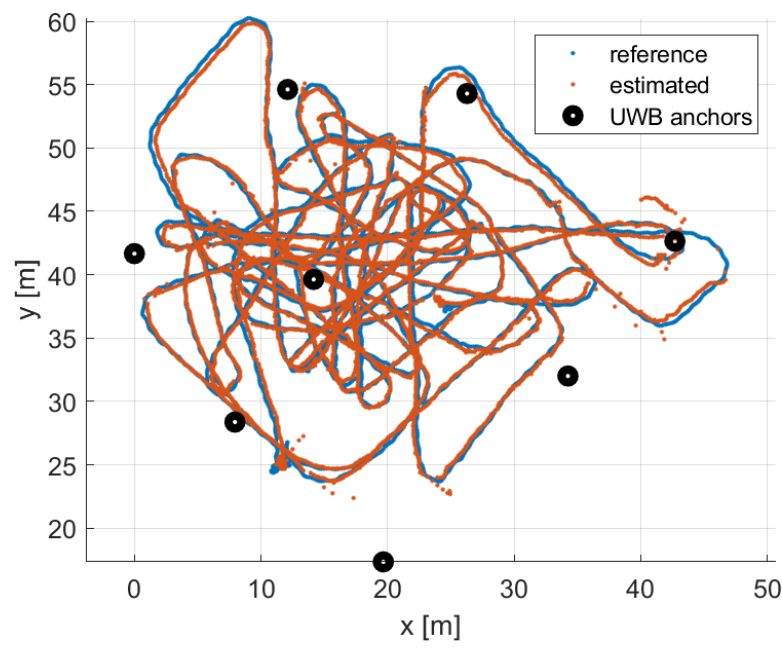

Figure 6. Example of pedestrian GNSS-based reference trajectory (blue) and estimated one (red).

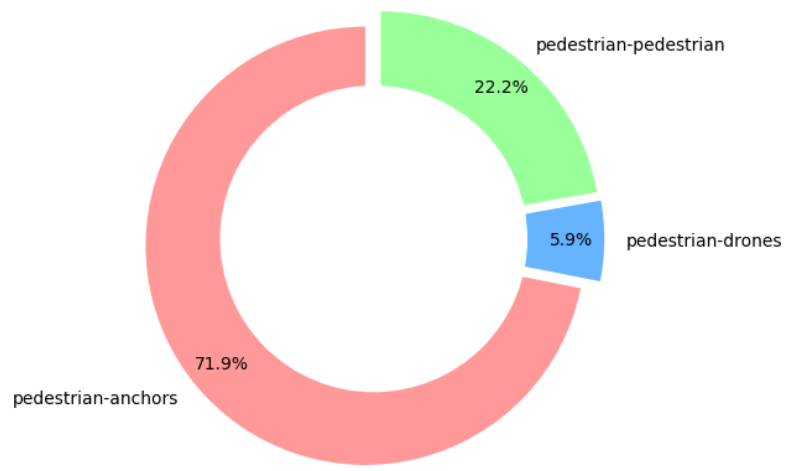

Figure 7. Distribution of the available UWB range measurements among the possible sources.

A collaborative approach can also have a positive impact on the UWB positioning performance, in particular for those devices which are not equipped of other reliable positioning systems. From this point of view, UWB pedestrian-to-pedestrian range measurements (P in Table 1) can play an important role, as they represent $22.2 \%$ of the overall collected UWB measurements, and their ranging success rate is higher than pedestrianto-anchors (A) and pedestrian-to-drones ( $\mathbf{D}$ in Table 1).

\begin{tabular}{c|c|c|c|c}
\hline & all & A & D & P \\
\hline success rate $\%$ & 54 & 59 & 19 & 72 \\
\hline
\end{tabular}

Table 1. UWB ranging success rate.

Nevertheless, the collaborative approach performance will be investigated in our future works.

Instead, the following figures summarize the results obtained by means of drone vision-based positioning.

For the sake of readability of the figures, the results shown in the following refer just to a two-minute part of the trajectories of the three pedestrians.

In particular, Fig. 8 shows the three pedestrian tracks, estimated by means of the vision-based approach, overlapped on the background image of the area monitored by the DJI Matrice

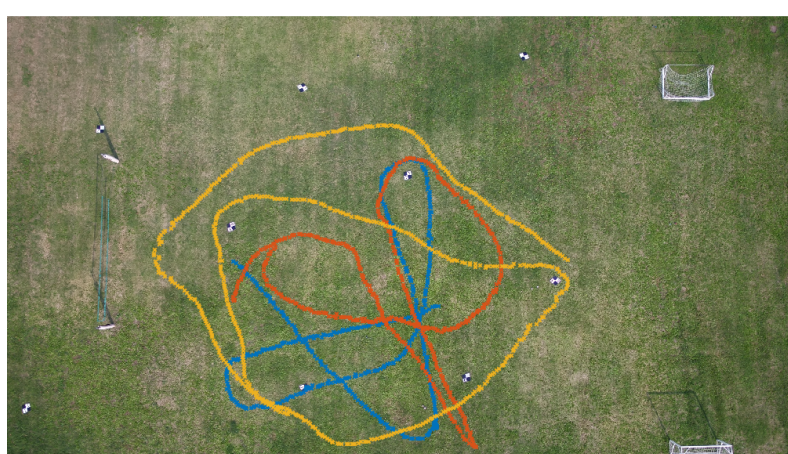

Figure 8. Drone vision-based tracks of the three pedestrians.

210 drone. The blue track corresponds to a portion of the track walked by pedestrian 1, which was already visible in Fig. 6 .

The three pedestrian tracks are also shown in Fig. 9, where the vision-based estimated (dot marks) are compared with the GNSS-based reference trajectories (solid lines).

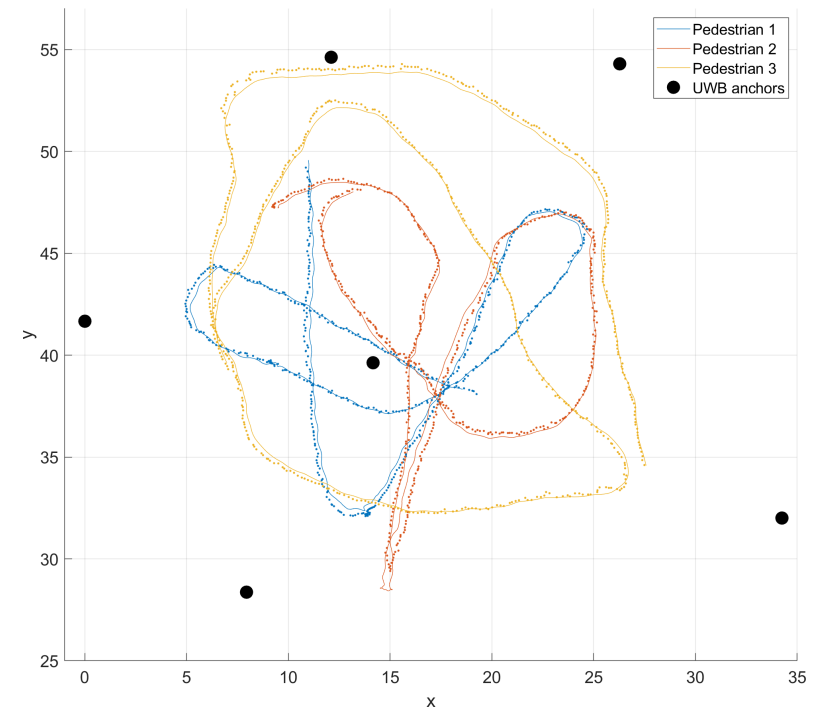

Figure 9. Drone vision-based tracks of the three pedestrians (dot marks) compared with their reference trajectories (solid lines, GNSS-based).

Then, the pedestrian 1 positioning error distribution, for the above mentioned two-minute dataset, is shown in Fig. 10 for the UWB approach, and in Fig. 11 for the vision-based method.

Finally, a comparison between the positioning error of the UWB and of the vision-based approach is shown in Fig. 12, where the values of two positioning errors are plotted for the considered two-minute dataset of pedestrian 1 . The corresponding numerical results are shown in Table 2

\begin{tabular}{c|c|c}
\hline & UWB & vision \\
\hline & 29 & 23 \\
Median err [cm] & 9 & 7 \\
Median absolute deviation err [cm] & 407 & 50 \\
\hline
\end{tabular}

Table 2. 2D positioning error for pedestrian 1 .

Given the obtained results, it is quite clear that the vision based approach provided in the considered dataset more stable results 


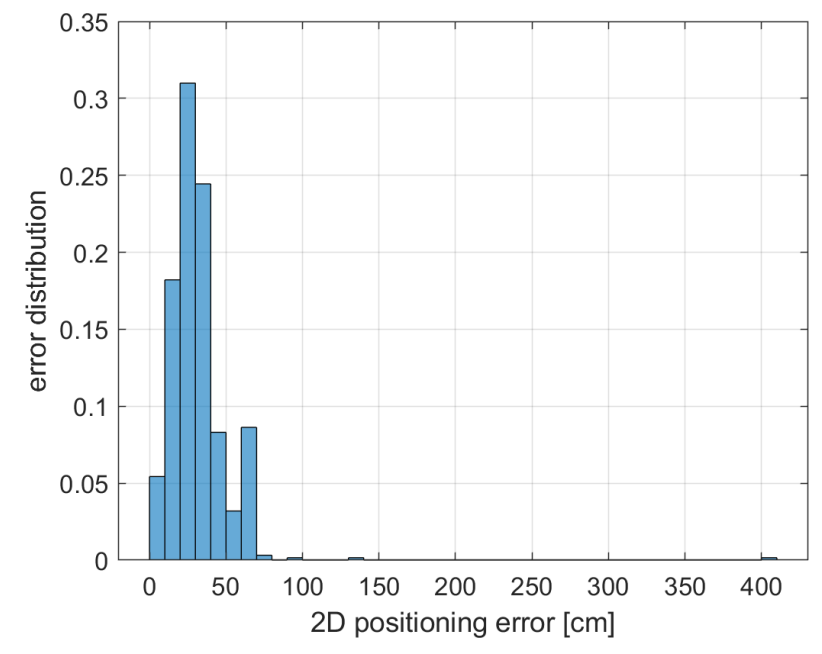

Figure 10. Static UWB infrastructure-based 2D positioning error distribution of pedestrian 1 .

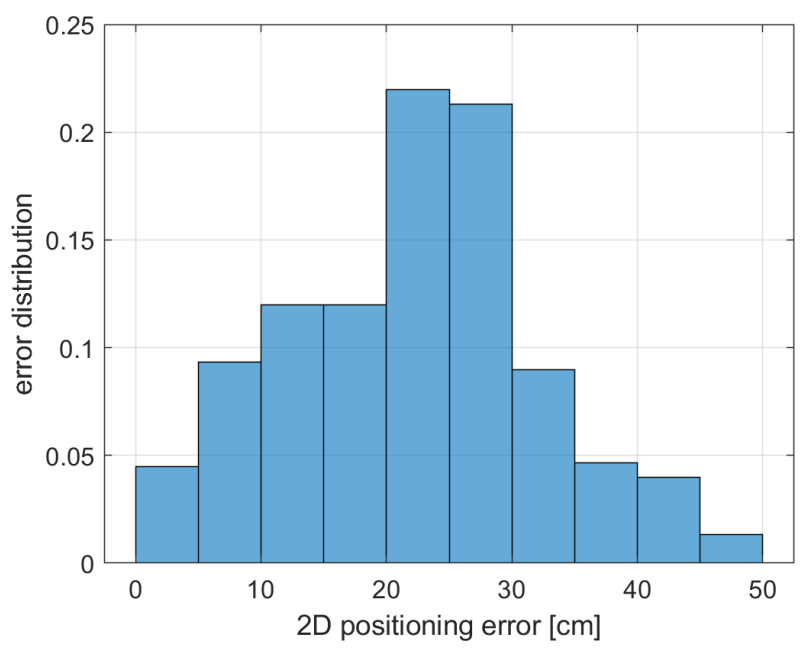

Figure 11. Drone vision-based 2D positioning error distribution of pedestrian 1 .

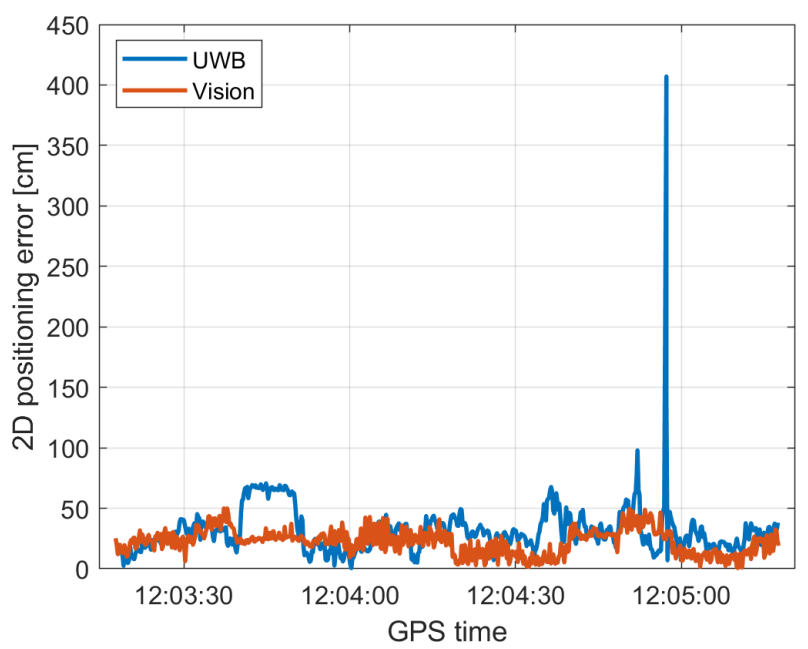

Figure 12. UWB vs drone-vision 2D positioning error for pedestrian 1.

with respect to the UWB approach, allowing in particular to significantly reduce the maximum error.
Nevertheless, the vision results did not provided any estimate for few time instants for pedestrian 2 , when he went outside of the area monitored by the drone, whereas the UWB system continuously provided estimates of his position.

Furthermore, when evaluating the vision results, it should also take into consideration that the vision approach aimed at estimating the centroid of the top view of the pedestrian, which typically did not correspond with the position of the GNSS antenna held by such person. In practice, such displacement shall also be considered for a more fair assessment of the vision-based performance.

The above considerations confirm that vision based approaches can provide very interesting positioning results, despite their fusion with other sensors, such as UWB, shall be considered in order to obtain more robust results.

\section{CONCLUSIONS}

This paper presented the comparison of two approaches, alternative to GNSS, for pedestrian positioning. Such two approaches were based on the use of UWB static infrastructure and on the analysis of video frames provided by a drone flying over the test area.

The UWB system performance was quite good (median 2D positioning error smaller than half a meter), however quite large errors may occasionally occur.

Vision, when usable, has shown to have a great potential in positioning systems, both stand-alone and combined with other sensors. In particular, the latter case shall lead to a more robust solution, which is clearly preferable when the goal is that of compensating for the unavailability of GNSS.

Our future works will deal with a more detailed analysis of the performance that can be obtained by integrating vision data with UWB, with the inertial sensor data, and also with GNSS raw measurements (Robustelli et al., 2019), and, in particular we will focus on the investigation of the performance of collaborative positioning approaches.

\section{REFERENCES}

Alinsavath, K. N., Nugroho, L. E., Hamamoto, K., 2019. The seamlessness of outdoor and indoor localization approaches based on a ubiquitous computing environment: A survey. Proceedings of the 2019 2nd International Conference on Information Science and Systems, 316-324.

Dabove, P., Di Pietra, V., 2019. Towards high accuracy GNSS real-time positioning with smartphones. Advances in Space Research, 63(1), 94-102.

de Groot, L., Infante, E., Jokinen, A., Kruger, B., Norman, L., 2018. Precise positioning for automotive with mass market gnss chipsets. Proceedings of the 31st International Technical Meeting of The Satellite Division of the Institute of Navigation (ION GNSS+ 2018), 596-610.

Emlid Ltd, 2019. Emlid docs. https://emlid.com/.

Fischler, M., Bolles, R., 1981. Random sample consensus: A paradigm for model fitting with applications to image analysis and automated cartography. Communications of the ACM, 24(6), 381-395. 
Gabela, J., Retscher, G., Goel, S., Perakis, H., Masiero, A., Toth, C., Gikas, V., Kealy, A., Koppányi, Z., Błaszczak-Bak, W. et al., 2019. Experimental evaluation of a UWB-based cooperative positioning system for pedestrians in GNSS-denied environment. Sensors, 19(23), 5274.

GSA, G., 2019. Market Report Issue 6. European Global Navigation Satellite Systems Agency.

He, F., Habib, A., 2016. Automated Relative Orientation of UAV-Based Imagery in the Presence of Prior Information for the Flight Trajectory. Photogrammetric Engineering \& Remote Sensing, 82(11), 879-891.

Hsu, L.-T., Gu, Y., Huang, Y., Kamijo, S., 2015. Urban pedestrian navigation using smartphone-based dead reckoning and 3D map-aided GNSS. IEEE Sensors Journal, 16(5), 1281-1293.

Leutenegger, S., Chli, M., Siegwart, R. Y., 2011. BRISK: Binary robust invariant scalable keypoints. Computer Vision (ICCV), 2011 IEEE International Conference on, IEEE, 25482555.

Luo, H., Li, Y., Wang, J., Weng, D., Ye, J., Hsu, L.-T., Chen, W., 2021. Integration of GNSS and BLE Technology With Inertial Sensors for Real-Time Positioning in Urban Environments. IEEE Access, 9, 15744-15763.

Pozyx Labs, 2015. Pozyx positioning system. https://www.pozyx.io/.

Retscher, G., Kealy, A., 2006. Ubiquitous positioning technologies for modern intelligent navigation systems. Geowissenschaftliche Mitteilungen, 57.
Retscher, G., Kealy, A., Gabela, J., Li, Y., Goel, S., Toth, C. K., Masiero, A., Błaszczak-Bak, W., Gikas, V., Perakis, H. et al., 2020. A benchmarking measurement campaign in GNSS-denied/challenged indoor/outdoor and transitional environments. Journal of Applied Geodesy, 14(2), 215-229.

Retscher, G., Leb, A., 2021. Development of a SmartphoneBased University Library Navigation and Information Service Employing Wi-Fi Location Fingerprinting. Sensors, 21(2), 432.

Robustelli, U., Baiocchi, V., Pugliano, G., 2019. Assessment of dual frequency GNSS observations from a Xiaomi Mi 8 Android smartphone and positioning performance analysis. Electronics, 8(1), 91.

Sakr, M., Masiero, A., El-Sheimy, N., 2020. LocSpeck: A Collaborative and Distributed Positioning System for Asymmetric Nodes Based on UWB Ad-Hoc Network and Wi-Fi Fingerprinting. Sensors, 20(1), 78.

Sturari, M., Liciotti, D., Pierdicca, R., Frontoni, E., Mancini, A., Contigiani, M., Zingaretti, P., 2016. Robust and affordable retail customer profiling by vision and radio beacon sensor fusion. Pattern Recognition Letters, 81, 30-40.

Tsai, G., Chen, Y., Chiang, K., Lai, Y., 2016. The Generation of Building Floor Plans Using Portable and Unmanned Aerial Vehicle Mapping Systems. ISPRS - The International Archives of Photogrammetry, Remote Sensing and Spatial Information Sciences, 41, 331.

Zeng, Q., Wang, J., Meng, Q., Zhang, X., Zeng, S., 2017. Seamless pedestrian navigation methodology optimized for indoor/outdoor detection. IEEE Sensors Journal, 18(1), 363-374. 